\title{
Multimodality Probes: Amphibian Cars for Molecular Imaging
}

$\mathbf{I}_{\mathbf{n}}$ day, PET/CT scanners rather than standalone PET scanners are being installed because it has been recognized that dual-modality imaging enhances the diagnostic information in clinical practice. Particularly when the images are acquired in a single imaging session, the diagnostic information of the integrated images is maximally exploited. The introduction of hybrid PET/CT scanners has extended the diagnostic power of ${ }^{18} \mathrm{~F}$-FDG PET in patients with cancer. The anatomic information allows more accurate delineation and characterization of suspected lesions. Because MRI has unmet soft-tissue

\section{See page 1371}

contrast and functional imaging capabilities, the introduction of hybrid MRI/PET scanners offers many advantages that go far beyond simply combining functional PET information with anatomic information. Particularly, the simultaneous acquisition of different functional parameters with PET in addition to high-resolution anatomic and functional information that can be obtained with MRI, MRS, and functional MRI would open yet another new era in diagnostic imaging.

For the development of hybrid MRI/ PET scanners, several technologic hurdles had to be overcome, especially because the photomultiplier tubes as

Received Apr. 22, 2008; revision accepted Apr. 24, 2008.

For correspondence or reprints contact: Otto C. Boerman, Department of Nuclear Medicine, Radboud University Nijmegen Medical Centre, P.O. Box 9101, 6500 HB Nijmegen, The Netherlands. E-mail: O.Boerman@nucmed.umcn.nl

COPYRIGHT @ 2008 by the Society of Nuclear Medicine, Inc.

DOI: 10.2967/jnumed.108.052274 applied in conventional PET scanners are incompatible with the high magnetic fields applied in MRI scanners. In particular, the development of detectors that are insensitive to magnetic fields, so-called avalanche photodiodes, proved to be an effective approach to circumvent the problem of incompatibility (1). Recently, a few prototypes of hybrid PET/MRI scanners have been developed (2-5).

The development of this new generation of hybrid scanners combining MRI and PET drives the development of new radiotracers and contrast agents. The functional information of PET relies on the choice of the tracer, whereas the functional and anatomic information of MRI can be further improved by applying paramagnetic contrast agents. In this issue of The Journal of Nuclear Medicine, Lee et al. describe the synthesis and characterization of a new probe that provides a signal both in PET and in MRI (6). To this end, the authors prepared iron oxide (IO) containing 5-nm nanoparticles that were coated with cyclic arginine-glycine-aspartic (RGD) peptides and with 1,4,7,10tetraazacyclododecane- $N, N^{\prime}, N^{\prime \prime}, N^{\prime \prime \prime}$,tetraacetic acid (DOTA), allowing labeling with the positron emitter ${ }^{64} \mathrm{Cu}(6)$. Their studies showed that these particles specifically localize in $\alpha_{\mathrm{v}} \beta_{3}$-expressing tumors in nude mice and thus can be used to visualize these tumors with smallanimal PET and with MRI.

The synthesis of this multimodality probe was designed very elegantly. Polyaspartic acid (PASP)-coated IO particles were coated with the cyclic RGD peptide via a poly(ethylene glycol) (PEG) spacer and with DOTA. The resulting RGD- and DOTA-coated IO 5-nm nanoparticles showed excellent magnetic properties for MRI and could be efficiently labeled with ${ }^{64} \mathrm{Cu}$ for PET. In vitro specific binding of these nanoparticles to $\alpha_{\mathrm{v}} \beta_{3}$ integrin-expressing cells was demonstrated, although it can be argued that it is not valid to report the $50 \%$ inhibitory concentration for nanoparticles, as the concentration of a suspension of nanoparticles cannot be compared with that of a peptide. On intravenous injection into nude mice with subcutaneous $\alpha_{v} \beta_{3}$-expressing glioma xenografts, specific accumulation of the nanoparticles in the tumor was observed with both small-animal PET and MRI. Although in this study 2 sets of animals were imaged on separate devices, these studies demonstrate that this new agent can be used as a contrast agent for MRI and as a specific tracer in PET.

The fundamental question is whether we require such multimodality probes for multimodality imaging. In hybrid PET/CT scanners, generally 2 independent agents are applied: patients receive a tracer for PET and in the same procedure a contrast agent is injected to enhance CT. PET provides the functional information, whereas contrast-enhanced CT adds the anatomic information. For PET/MRI, one could select the MRI contrast agent and the PET tracer. Both images could then provide complementary information, tailored to the clinical or research question to be answered. Using an integrated probe for both imaging modalities could save an intravenous injection, but the added value of a combined probe as compared with 2 independent probes appears limited as such a combined tracer is always designed as a compromise. The probe should have optimal characteristics for MRI while also having optimal characteristics as a PET tracer. Although PET is an extremely sensitive imaging modality, for contrast in MRI relatively high amounts of the agent are required. The mass of IO in the current probe 


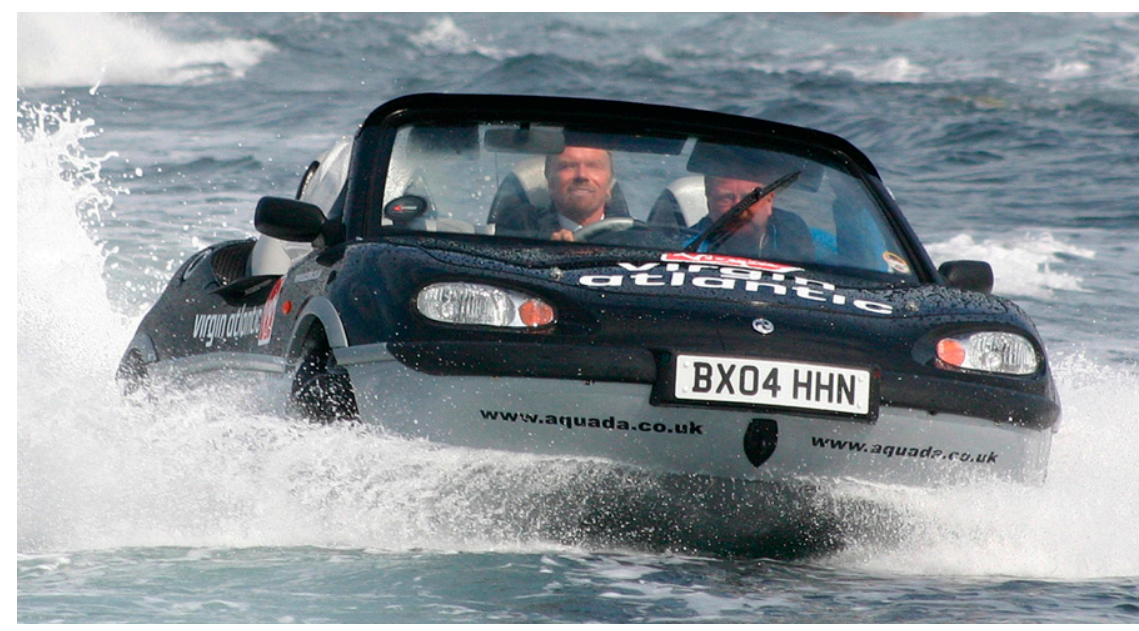

Image courtesy of Gibbs Technologies Media Centre.

limits the PET characteristics of the tracer and vice versa; by using a combined probe, the unique sensitivity of PET is not fully exploited.

In their article Lee et al. (6) show that the in vivo characteristics for PET with ${ }^{64} \mathrm{Cu}$-labeled RGD-coated IO nanoparticles are suboptimal compared with those of ${ }^{64} \mathrm{Cu}$-DOTA-RGD as developed previously by the same group (7). To visualize the nanoparticles with MRI, more than $300 \mu \mathrm{g}$ of the nanoparticles per mouse are required, whereas for PET only $1 \mu \mathrm{g}$ of ${ }^{64} \mathrm{Cu}$-labeled DOTA-RGD peptide is injected. Because of the enhanced circulatory half-life of the nanoparticles compared with the half-life of

the RGD peptide, the nonspecific uptake of the nanoparticles in the tumor is 10fold higher: 2-3 percentage injected dose per gram $(\% \mathrm{ID} / \mathrm{g})$ versus $0.2 \% \mathrm{ID} / \mathrm{g}$. Similarly, because of the affinity of the nanoparticles for the reticuloendothelial system, uptake in the liver was 10-fold higher: $31 \% \mathrm{ID} / \mathrm{g}$ versus $3 \% \mathrm{ID} / \mathrm{g}(6)$. These data underline that this combinedmodality probe is not optimal for PET.

Multimodality imaging benefits from the complementary information provided by the imaging modalities, whereas the use of a combined probe poses the challenge of demonstrating optimal imaging characteristics without compromising the information ob- tained with both modalities. In a way, the development of a multimodality probe bears the same type of inherent risk as that in designing an amphibious car: on the road it is not as fast as a sports car, and in the water it does not perform as a speedboat.

Otto C. Boerman Wim J.G. Oyen

Radboud University Nijmegen Medical Centre Nijmegen, The Netherlands

\section{REFERENCES}

1. Judenhofer MS, Wehrl HF, Newport DF, et al. Simultaneous PET-MRI: a new approach for functional and morphological imaging. Nat Med. 2008;14: 459-465.
2. Shao Y, Cherry SR, Farahani K, Slates R, Silverman RW, Meadors K. Development of a PET detector system compatible with MRI/NMR systems. IEEE Trans Nucl Sci. 1997;44:1167-1171.

3. Marsden PK, Strul D, Keevil SF, Williams SC, Cash D. Simultaneous PET and NMR. Br J Radiol. 2002; 75(suppl):S53-S59.

4. Lucignani G. Time-of-flight PET and PET/MRI: recurrent dreams or actual realities? Eur J Nucl Med Mol Imaging. 2006;33:969-971.

5. Pichler BJ, Wehrl HF, Kolb A, Judenhofer MS. Positron emission tomography/magnetic resonance imaging: the next generation of multimodality imaging? Semin Nucl Med. 2008;38:199-208.

6. Lee H-Y, Li Z, Chen K, et al. PET/MRI dualmodality tumor imaging using arginine-glycineaspartic (RGD)-conjugated radiolabeled iron oxide nanoparticles. J Nucl Med. 2008;49:1371-1379.

7. Chen X, Hou Y, Tohme M, et al. Pegylated arggly-asp peptide: ${ }^{64} \mathrm{Cu}$ labeling and PET imaging of brain tumor alphavbeta3-integrin expression. $\mathrm{J} \mathrm{Nucl}$ Med. 2004;45:1776-1783. 